\title{
ON FINITE ADDITIVE 2-BASES
}

\author{
LAURENT HABSIEGER
}

\begin{abstract}
For a positive integer $N$, a set $\mathcal{B}$ of integers from $\{0,1, \ldots, N-1\}$ is called an additive 2-basis for $N$ if every integer $n \in\{0,1, \ldots, N-1\}$ may be represented as the sum of 2 elements of $\mathcal{B}$. We discuss the methods used to estimate the minimal size of an additive 2-basis for $N$. We provide new examples to enrich this survey, which give good bounds. For instance, we slightly improve on the current record, from 0.46972 to 0.46906 .
\end{abstract}

\section{INTRODUCTION}

A 2-basis for $N$ is a set $\mathcal{B}$ of integers in $[0, N-1]$ such that every nonnegative integer up to $N-1$ may be written as the sum of two elements in $\mathcal{B}$. It is quite natural to ask for $\mathcal{B}$ to be of minimal size. The exact asymptotic behaviour of this minimal cardinality is still unknown. By enumerating sums of either distinct elements or identical elements, we get the inequality

$$
\left(\begin{array}{c}
|\mathcal{B}| \\
2
\end{array}\right)+|\mathcal{B}| \geq N
$$

that is, $|\mathcal{B}|^{2}+|\mathcal{B}| \geq 2 N$. We shall study

$$
\sigma=\limsup _{N \rightarrow \infty} \frac{N}{|B|^{2}}
$$

and we already know the trivial estimate $\sigma \leq 1 / 2$. The best construction of a 2-basis was found by Mrose [6] and gives the lower bound $\sigma \geq 2 / 7=0.285714 \ldots$. Numerous authors have found small improvements on the upper bound:

$\begin{array}{lcl}0.4992 & \text { Rohrbach [8] } & 1937 \\ 0.4903 & \text { Moser [4] } & 1960 \\ 0.4867 & \text { Riddell [7] } & 1960 \\ 0.4847 & \text { Moser, Pounder, Riddell [5] } & 1969 \\ 0.4802 & \text { Klotz 3] } & 1969 \\ 0.4789 & \text { Güntürk, Nathanson 2] } & 2006 \\ 0.4775 & \text { An-Ping [1] } & 2012 \\ 0.46972 & \text { Yu [9] } & 2008\end{array}$

Li An-Ping was unaware of the results in [3, 9], and extended Klotz' approach [4]; that is why we included his result in the list. The paper needs some fixing, and this is done in section 4 .

Received by the editors April 15, 2013.

2010 Mathematics Subject Classification. Primary 11B13; Secondary 11B34.

Key words and phrases. Additive bases.

This work was supported by the French National Agency for Research (CAESAR ANR-12BS01-0011). The author also thanks Alain Plagne and Victor Lambert for their careful reading of a preliminary version of this paper. 
All the methods used after Rohrbach's result involve Fourier series, in some way or another. In section 2 we shall show that the only use of generalized Fourier series is not sufficient to get interesting results. So the methods are always combined with an extra idea that allows to get a nontrivial bound. We distinguish four methods, namely:

i) the use of a basic combinatorial lemma;

ii) refining this combinatorial lemma;

iii) using double Fourier series;

iv) using periods greater than one.

Each of these methods will be described in sections 3-6, together with numerous examples. Some new examples give better results than the ones in the literature. For instance, we improve on the best current bound, as shown in the following table.

$\begin{array}{ccc}\text { Method } & \text { Previous results } & \text { New results } \\ \text { Combinatorial lemma } & 0.4903[\underline{4} & 0.4843 \text { [Ex. 3.3] } \\ & 0.4867[7] & 0.4827 \text { [Ex. 3.4] } \\ & 0.4847[\underline{5}] & \\ \text { Refined combinatorial lemma } & 0.4802[3] & 0.47737 \text { [Sect. 4] } \\ & 0.4775[\underline{1]} & \\ \text { Double Fourier series } & 0.4789[2] & 0.47873 \text { [Sect. 5] } \\ \text { Periods greater than one } & 0.46972[9] & 0.46906 \text { [Ex. 6.3] }\end{array}$

\section{A general approach}

Assume $\mathcal{B}$ is an additive 2-basis for $N$. We shall use several auxiliary functions, namely the (complex) generating function $f_{\mathcal{B}}$, the representation function $r_{\mathcal{B}}(n)$ and the difference function $d_{\mathcal{B}}(n)$. More precisely define

$$
\begin{aligned}
f_{\mathcal{B}}(t) & =\sum_{b \in \mathcal{B}} \exp (2 i \pi b t), \\
r_{\mathcal{B}}(n) & =\#\left\{\left(b_{1}, b_{2}\right) \in \mathcal{B}^{2}: b_{1}+b_{2}=n\right\} \\
d_{\mathcal{B}}(n) & =\#\left\{\left(b_{1}, b_{2}\right) \in \mathcal{B}^{2}: b_{1}-b_{2}=n\right\} .
\end{aligned}
$$

Note that $f_{\mathcal{B}}$ is a 1 -periodic function with $f_{\mathcal{B}}(0)=|\mathcal{B}|$. Let us try to estimate it:

$$
f_{\mathcal{B}}(t)^{2}=\sum_{n=0}^{2 N-2} r_{\mathcal{B}}(n) \exp (2 i \pi n t)=2 \sum_{m=0}^{N-1} \exp (2 i \pi m t)+\sum_{m=0}^{2 N-2} r_{\mathcal{B}}^{*}(m) \exp (2 i \pi m t),
$$

with

$$
r_{\mathcal{B}}^{*}(m)= \begin{cases}r_{\mathcal{B}}(m)-2 & \text { if } 0 \leq m \leq N-1 \\ r_{\mathcal{B}}(m) & \text { otherwise }\end{cases}
$$

Since $\mathcal{B}$ is a 2 -basis for $N$, we get

$$
r_{\mathcal{B}}^{*}(m) \geq \begin{cases}-1 & \text { if } m \in 2 \cdot \mathcal{B} \text { and } 0 \leq m \leq N-1 \\ 0 & \text { otherwise }\end{cases}
$$


This implies that

$$
\begin{aligned}
M^{2}:=\sum_{m=0}^{2 N-2}\left|r_{\mathcal{B}}^{*}(m)\right| \leq \sum_{m=0}^{2 N-2} r_{\mathcal{B}}^{*}(m)+2|\mathcal{B}| & =\sum_{m=0}^{2 N-2} r_{\mathcal{B}}(m)-2 N+2|\mathcal{B}| \\
& =|\mathcal{B}|^{2}-2 N+2|\mathcal{B}| .
\end{aligned}
$$

We can use this last property to upper bound the generating function on the unit circle.

Lemma 2.1. For $|t| \leq 1 / 2$, we have

$$
\left|f_{\mathcal{B}}(t)^{2}-2 N \int_{0}^{1} \exp (2 i \pi N t x) d x\right| \leq|\mathcal{B}|^{2}-2 N+2|\mathcal{B}|+2 \pi \text {. }
$$

Proof. The inequality is trivial for $t=0$. For $t \neq 0$, we extend the proof of [9. Lemma 2.2]. We use (2.1) and (2.3) to obtain

$$
\left|f_{\mathcal{B}}(t)^{2}-2 \frac{1-\exp (2 i \pi N t)}{1-\exp (2 i \pi t)}\right| \leq \sum_{m=0}^{2 N}\left|r_{\mathcal{B}}^{*}(m)\right| \leq|B|^{2}-2 N+2|B| .
$$

We easily check that, for $|x| \leq \pi$,

$$
\left|\frac{1}{1-\exp (i x)}+\frac{1}{i x}\right|=\left|\frac{\int_{0}^{x}(x-t) \exp (i t) d t}{x \sin (x / 2)}\right| \leq \frac{\int_{0}^{x}(x-t) d t}{x^{2} / \pi}=\frac{\pi}{2},
$$

which implies the inequalities

$$
\left|\frac{1-\exp (2 i \pi N t)}{1-\exp (2 i \pi t)}-N \int_{0}^{1} \exp (2 i \pi N t x) d x\right| \leq \frac{\pi}{2}|1-\exp (2 i \pi N t)| \leq \pi .
$$

The lemma then easily follows.

Define $\mathcal{W}$ the set of functions $w$ such that:

i) there exists a sequence of real numbers $\left(t_{k}\right)_{k \in \mathbb{Z}}$ with $t_{0}=0$,

ii) there exists a sequence of complex numbers $(\widehat{w}(k))_{k \in \mathbb{Z}}$ with

$$
\|w\|:=\sum_{k \in \mathbb{Z}}|\widehat{w}(k)|<\infty,
$$

iii) for $x$ real, we have $w(x)=\sum_{k \in \mathbb{Z}} \widehat{w}(k) \exp \left(2 i \pi t_{k} x\right)$.

Note that condition ii) implies that $w$ is a continuous function. If all the generalized Fourier coefficients $\widehat{w}(k)$ are nonnegative, we remark that $\|w\|=w(0)$.

When $x$ is replaced by $x+y$, we get a similar expansion for $w(x+y)$, with $\widehat{w}(k)$ replaced by $\widehat{w}(k) \exp \left(2 i \pi t_{k} y\right)$. This shows that both $\widehat{w}(0)$ and $\|w\|$ are invariant by variable-translation.

For $w \in \mathcal{W}$, define

$$
R_{\mathcal{B}}(w)=\sum_{n=0}^{2 N-2} w(n / N) r_{\mathcal{B}}(n) \quad \text { and } \quad D_{\mathcal{B}}(w)=\sum_{n=-N+1}^{N-1} w(n / N) d_{\mathcal{B}}(n) .
$$

Using the expansion of $w$, we obtain

$$
R_{\mathcal{B}}(w)=\sum_{k \in \mathbb{Z}} \widehat{w}(k) \sum_{n=0}^{2 N-2} r_{\mathcal{B}}(n) \exp \left(2 i \pi t_{k} n / N\right)=\sum_{k \in \mathbb{Z}} \widehat{w}(k) f_{\mathcal{B}}\left(\frac{t_{k}}{N}\right)^{2},
$$


and similarly

$$
D_{\mathcal{B}}(w)=\sum_{k \in \mathbb{Z}} \widehat{w}(k)\left|f_{\mathcal{B}}\left(\frac{t_{k}}{N}\right)\right|^{2} .
$$

Lemma 2.2. For $w \in \mathcal{W}$, we have

$$
\left|R_{\mathcal{B}}(w)-2 N \int_{0}^{1} w(x) d x\right| \leq\|w\|\left(|\mathcal{B}|^{2}-2 N+2|\mathcal{B}|+2 \pi\right) .
$$

Proof. By the definition of $w$ and (2.4), we have

$$
\begin{aligned}
R_{\mathcal{B}}(w)-2 N \int_{0}^{1} w(x) d x & =R_{\mathcal{B}}(w)-2 N \sum_{k \in \mathbb{Z}} \widehat{w}(k) \int_{0}^{1} \exp \left(2 i \pi t_{k} x\right) d x \\
& =\sum_{k \in \mathbb{Z}} \widehat{w}(k)\left(f_{\mathcal{B}}\left(\frac{t_{k}}{N}\right)^{2}-2 N \int_{0}^{1} \exp \left(2 i \pi t_{k} x\right) d x\right),
\end{aligned}
$$

from which we get

$$
\left|R_{\mathcal{B}}(w)-2 N \int_{0}^{1} w(x) d x\right| \leq \sum_{k \in \mathbb{Z}}|\widehat{w}(k)|\left(|\mathcal{B}|^{2}-2 N+2|\mathcal{B}|+2 \pi\right),
$$

by Lemma 2.1. The lemma then easily follows from the definition of $\|w\|$.

Lemma 2.3. For $w \in \mathcal{W}$, we get

$$
\begin{aligned}
\left(\min _{x \in[0,2]} w(x)+o(1)\right) & \left(|\mathcal{B}|^{2}-2 N\right) \\
\leq & R_{\mathcal{B}}(w)-2 N \int_{0}^{1} w(x) d x \leq\left(\max _{x \in[0,2]} w(x)\right)+o(1)\left(|\mathcal{B}|^{2}-2 N\right) .
\end{aligned}
$$

Proof. By the definition of $r_{\mathcal{B}}^{*}(n)$ and $(2.2)$, we obtain

$$
R_{\mathcal{B}}(w)-2 \sum_{n=0}^{N-1} w(n / N)=\sum_{n=0}^{2 N-2} w(n / N) r_{\mathcal{B}}^{*}(n)=\sum_{n=0}^{2 N-2} w(n / N)\left|r_{\mathcal{B}}^{*}(n)\right|+O(|\mathcal{B}|)
$$

By (2.3) we get

$$
\begin{aligned}
\min _{x \in[0,2]} w(x)\left(|\mathcal{B}|^{2}-2 N\right)+O(|\mathcal{B}|) & \\
& \leq R_{\mathcal{B}}(w)-2 \sum_{n=0}^{N-1} w(n / N) \leq \max _{x \in[0,2]} w(x)\left(|\mathcal{B}|^{2}-2 N\right)+O(|\mathcal{B}|) .
\end{aligned}
$$

Since $w$ is continuous, we have

$$
\sum_{n=0}^{N-1} w(n / N)=N \int_{0}^{1} w(x) d x+o(N)
$$

and the lemma follows.

We see from Lemmas 2.2 and 2.3 that this way to use the generalized Fourier series is not sufficient to get any information on the size of $\mathcal{B}$ : once divided by $|\mathcal{B}|^{2}-2 N$, these inequalities only involve $w$, and don't provide any information on $|\mathcal{B}|$. We therefore need extra tools, and we shall investigate several possible ones in the following sections. 
In view of Lemma 2.1 , it is natural to pay special attention to the values $t=$ $1 / N, 2 / N, \ldots, 1-1 / N$, since the integral vanishes in this case. By (2.4) we are thus led to study the usual Fourier series, namely those for which $t_{k}=k$. This will be done in the next three sections, while the fourth forthcoming section will be devoted to the more general case $t_{k}=k / p$. Other choices of the sequence $\left(t_{k}\right)$ could be interesting, but it seems difficult to get accurate estimates then.

\section{USing A COMBINATORIAL LEMMA}

Let us consider the case $t_{k}=k$, so that

$$
f_{\mathcal{B}}\left(\frac{k}{N}\right)^{2}=\sum_{m=0}^{2 N-2} r_{\mathcal{B}}^{*}(m) \exp \left(2 i \pi m \frac{k}{N}\right)
$$

for $N \nmid k$. In this special case, we can deduce an upper bound from (2.3):

$$
\left|f_{\mathcal{B}}\left(\frac{k}{N}\right)\right| \leq M
$$

for $N \nmid k$, while $f_{\mathcal{B}}(k / N)=|\mathcal{B}|$ for $N \mid k$.

Let us introduce an auxiliary function $\varphi$ defined by

$$
\varphi(t)=\sum_{k \in \mathbb{Z}} \widehat{\varphi}(k) \exp (2 i \pi k t)
$$

with $\|\varphi\|=\sum_{k}|\widehat{\varphi}(k)|<\infty$. We therefore have

$$
S:=\sum_{b \in \mathcal{B}} \varphi\left(\frac{b}{N}\right)=\sum_{k \in \mathbb{Z}} \widehat{\varphi}(k) f_{\mathcal{B}}\left(\frac{k}{N}\right) .
$$

Put $\ell=\# \mathcal{B} \cap[N / 2, N-1]$. Moser's combinatorial lemma [5] may be stated as follows.

Lemma 3.1. We have $\ell \leq M$, which gives

$$
\# \mathcal{B} \cap[N / 2, N-1] \leq \sqrt{|\mathcal{B}|^{2}-2 N+2|\mathcal{B}|} .
$$

Proof. The sum of any pair of elements in $\mathcal{B} \cap[N / 2, N-1]$ will give an element in $(\mathcal{B}+\mathcal{B}) \cap[N, 2 N-2]$. We thus get

$$
\ell^{2} \leq \sum_{m=N}^{2 N-2} r_{\mathcal{B}}(m)=\sum_{m=N}^{2 N-2} r_{\mathcal{B}}^{*}(m) \leq \sum_{m=0}^{2 N-2}\left|r_{\mathcal{B}}^{*}(m)\right|=M^{2},
$$

and the lemma is proved.

Let us use the double expression for $S$ given in (3.2). By (3.1) we have

$$
(|\mathcal{B}|-\ell) \min _{[0,1 / 2]} \varphi+\ell \min _{[1 / 2,1]} \varphi \leq S \leq M \sum_{N \nmid k}|\widehat{\varphi}(k)|+|\mathcal{B}|\left(\widehat{\varphi}(0)+\sum_{\substack{N \mid k \\ k \neq 0}} \widehat{\varphi}(k)\right) .
$$

In order to use Lemma 3.1, we need to assume that $\min _{[0,1 / 2]} \varphi \geq \min _{[1 / 2,1]} \varphi$. We then get from (3.3)

$$
S \geq|\mathcal{B}| \min _{[0,1 / 2]} \varphi-M\left(\min _{[0,1 / 2]} \varphi-\min _{[1 / 2,1]} \varphi\right) .
$$


From $\|\varphi\|<\infty$ we deduce

$$
\sum_{\substack{N \mid k \\ k \neq 0}}|\widehat{\varphi}(k)|=o(1)
$$

By (3.3) this implies

$$
S \leq M(\|\varphi\|-|\widehat{\varphi}(0)|)+|\mathcal{B}| \widehat{\varphi}(0)+o(|\mathcal{B}|) .
$$

Combining (3.4) and (3.5) leads to the nontrivial inequality

$$
|\mathcal{B}|\left(\min _{[0,1 / 2]} \varphi-\widehat{\varphi}(0)+o(1)\right) \leq M\left(\|\varphi\|-|\widehat{\varphi}(0)|+\min _{[0,1 / 2]} \varphi-\min _{[1 / 2,1]} \varphi\right) .
$$

Note that the factor of $M$ is positive when $\varphi$ is nonconstant. By (2.3), we know that $M \leq \sqrt{|\mathcal{B}|^{2}-2 N+O(|\mathcal{B}|)}$. Plugging in (3.6) this estimate leads to an inequality involving only $|\mathcal{B}|, N$ and $\varphi$ :

$$
\min _{[0,1 / 2]} \varphi-\widehat{\varphi}(0)+o(1) \leq \sqrt{1-\frac{2 N}{|\mathcal{B}|^{2}}}\left(\|\varphi\|-|\widehat{\varphi}(0)|+\min _{[0,1 / 2]} \varphi-\min _{[1 / 2,1]} \varphi\right) .
$$

In order to square (3.7), we ask for the condition $\min _{[0,1 / 2]} \varphi>\widehat{\varphi}(0)$ to be satisfied. Since $2 \widehat{\varphi}(0) \geq \min _{[0,1 / 2]} \varphi+\min _{[1 / 2,1]} \varphi$, this last condition implies the earliest condition, $\min _{[0,1 / 2]} \varphi \geq \min _{[1 / 2,1]} \varphi$. We thus deduce the following theorem.

Theorem 3.2. For $\min _{[0,1 / 2]} \varphi-\widehat{\varphi}(0) \geq 0$, we have

$$
\sigma \leq \frac{1}{2}\left(1-\left(\frac{\min _{[0,1 / 2]} \varphi-\widehat{\varphi}(0)}{\|\varphi\|-|\widehat{\varphi}(0)|+\min _{[0,1 / 2]} \varphi-\min _{[1 / 2,1]} \varphi}\right)^{2}\right) .
$$

If $\varphi$ is changed into $\varphi+C$, for some constant $C$, the differences $\min _{[0,1 / 2]} \varphi-\widehat{\varphi}(0)$, $\|\varphi\|-|\widehat{\varphi}(0)|$ and $\min _{[0,1 / 2]} \varphi-\min _{[1 / 2,1]} \varphi$ remain invariant. So the bound given in the theorem is also invariant when $\varphi$ is replaced by $A \varphi+B$, for any constants $A$ and $B>0$. People often consider the case $\widehat{\varphi}(0)=0$, but this is only for convenience.

We shall illustrate this theorem with several examples. It would be interesting to find the best possible constant obtained this way, but this seems to be a very difficult task.

Example $3.1([5])$. Take

$$
\varphi(t)=\sin (2 \pi t)+\frac{\cos (4 \pi t)}{2}=\frac{1}{2}+(1-\sin (\pi t)) \sin (\pi t) .
$$

We have $\widehat{\varphi}(0)=0,\|\varphi\|=3 / 2, \min _{[0,1 / 2]} \varphi=1 / 2$ and $\min _{[1 / 2,1]} \varphi=-3 / 2$, which gives the upper bound $\sigma \leq 24 / 49<0.4898$.

Example $3.2(\underline{6})$. Define

$$
\varphi(t)= \begin{cases}1 & \text { if } t \in[0,1 / 2] \\ 1+\pi \sin (2 \pi t) & \text { if } t \in[1 / 2,1]\end{cases}
$$

We find

$$
\widehat{\varphi}(k)= \begin{cases}\frac{\pi}{4 i k} & \text { if } k= \pm 1 \\ \frac{2}{k^{2}-1} & \text { if } k \text { is even, } k \neq 0 \\ 0 & \text { otherwise. }\end{cases}
$$


We thus get $\|\varphi\|=\varphi(0)+\pi / 2=1+\pi / 2$. By construction we also have $\min _{[0,1 / 2]} \varphi=$ 1 and $\min _{[1 / 2,1]} \varphi=1-\pi$. Applying Theorem 3.2 gives

$$
\sigma \leq \frac{1}{2}\left(1-\left(1+\frac{3 \pi}{2}\right)^{-2}\right)<0.4847 .
$$

Another natural function to consider is given in the next example.

Example 3.3. Consider

$$
\varphi(t)= \begin{cases}0 & \text { if } t \in[0,1 / 2] \\ (x-1 / 2)(x-1) & \text { if } t \in[1 / 2,1] .\end{cases}
$$

We easily find $\min _{[0,1 / 2]} \varphi=0$ and $\min _{[1 / 2,1]} \varphi=-1 / 16$. The Fourier coefficients are given by

$$
\widehat{\varphi}(n)= \begin{cases}-1 / 48 & \text { if } n=0 \\ \frac{1}{4 \pi^{2} n^{2}} & \text { if } n \neq 0 \text { is even } \\ \frac{i}{2 \pi^{3} n^{3}} & \text { if } n \text { is odd. }\end{cases}
$$

We thus get

$$
\|\varphi\|-|\widehat{\varphi}(0)|=\frac{1}{48}+\frac{7 \zeta(3)}{8 \pi^{3}}=0.05475549 \ldots .
$$

We obtain this way that $\sigma<0.4843$.

Let us give our best example now, whose form was motivated by the study in section 6 .

Example 3.4. Consider

$$
\varphi(t)= \begin{cases}0 & \text { if } t \in[0,1 / 2] \\ (1 / 2-x) / \delta & \text { if } t \in[1 / 2,1 / 2+\delta], \\ -1 & \text { if } t \in[1 / 2+\delta, 1-\delta] \\ (x-1) / \delta & \text { if } t \in[1-\delta, 1] .\end{cases}
$$

It is obvious that $\min _{[0,1 / 2]} \varphi=0$ and $\min _{[1 / 2,1]} \varphi=-1$. With the notation of section 6 , we have $\varphi=-w_{1,1 / 4-\delta, 1 / 4,1 / 4}$. Using the calculations detailed there, we get $\widehat{\varphi}(0)=-1 / 2+\delta$ and

$$
\|\varphi\|-|\widehat{\varphi}(0)|=\sum_{n=1}^{\infty} \frac{|\cos (2 \pi n(1 / 4-\delta))-\cos (\pi n / 2)|}{\pi^{2} \delta n^{2}} .
$$

The choice $\delta=1 / 14$ seems to be the best one, and leads to the better bound, $\sigma<0.4827$.

\section{USING A REFINED COMBINATORIAL LEMMA}

Klotz [3] and Li An-Ping [1] used the following lemma with $\theta=3 / 8$, a lemma that refines Lemma 3.1.

Lemma 4.1. For any $\theta \in[0,1 / 2]$, we have

$$
M^{2} \geq \ell^{2}+2 \ell_{1}(\theta) \ell_{2}(\theta),
$$

with $\ell_{1}(\theta)=\# \mathcal{B} \cap[\theta N, N / 2]$ and $\ell_{2}(\theta)=\# \mathcal{B} \cap[(1-\theta) N, N]$. 
Proof. We proceed as in the proof of Lemma 3.1 and look for elements in $\mathcal{B}+\mathcal{B}$ that are at least $N$. The sums $b_{1}+b_{2}$ and $b_{2}+b_{1}$, with $b_{1} \in B \cap[\theta N, N / 2]$ and $b_{2} \in \mathcal{B} \cap[(1-\theta) N, N]$, are not counted in the proof of Lemma 3.1. Thus we get

$$
\ell^{2}+2 \ell_{1}(\theta) \ell_{2}(\theta) \leq \sum_{m=N}^{2 N-2} r_{\mathcal{B}}(m) \leq M^{2}
$$

Klotz [3] used the function

$$
\varphi(t)= \begin{cases}1 & \text { if } t \in[0,1 / 4] \\ 3-8 x & \text { if } t \in[1 / 4,1 / 2] \\ -1 & \text { if } t \in[1 / 2,3 / 4] \\ 8 x-7 & \text { if } t \in[3 / 4,1]\end{cases}
$$

to get the inequality

$$
\ell_{1}(3 / 8) \geq \# \mathcal{B} \cap[0, N / 4]-\ell-\sqrt{2} M .
$$

He also considered the function given in Example 3.3 to obtain the lower bound

$$
\pi\left(1-\frac{1}{\sqrt{2}}\right) \ell_{2}(3 / 8) \geq|\mathcal{B}|-\frac{\pi}{\sqrt{2}} \ell-\left(1+\frac{\pi}{2}\right) M .
$$

He then proved the bound $\sigma \leq 0.4802$ by combining Lemma 4.1 with (4.1) and (4.2), and using the fact that $\mathcal{B} \cap[0, N / 4]$ is a 2-base for $N / 4$.

Li An-Ping [1] studied three other auxiliary functions to relate $\ell, \ell_{1}(3 / 8)$ and $\ell_{2}(3 / 8)$ to $|B|$ and $M$. More precisely, he stated the three inequalities

$$
\begin{aligned}
\ell_{1}(3 / 8) & \geq \frac{15}{16}|\mathcal{B}|-2.1 M-2 \ell+o(1), \\
\frac{4 \pi}{3} \ell_{2}(3 / 8) & \geq|\mathcal{B}|-3.893 M+o(1), \\
\ell_{2}(3 / 8) & \geq \frac{11}{16}|\mathcal{B}|-2.148 M-\ell+o(1) .
\end{aligned}
$$

Let us describe how to get these. We can also try to refine these inequalities by studying other examples similar to those from Section 3. We checked numerous examples, but only one allowed us to improve on Li An-Ping's result.

Example 4.1. The inequality (4.3) is obtained by studying the function $\varphi=$ $-w_{1,5 / 32,9 / 32,9 / 32}-\frac{1}{2} w_{1,0,1 / 16,9 / 16}$ with the notation of section 6 . Using the calculations detailed there, we get $\widehat{\varphi}(0)=-15 / 32$ and

$$
\begin{aligned}
\widehat{\varphi}(n)=\frac{4}{\pi^{2} n^{2}} \exp \left(\frac{9 i \pi n}{16}\right)\left(\cos \left(\frac{9 \pi n}{16}\right)-\cos \left(\frac{5 \pi n}{16}\right)\right) & \\
& +\frac{4}{\pi^{2} n^{2}} \exp \left(\frac{9 i \pi n}{8}\right)\left(\cos \left(\frac{\pi n}{8}\right)-1\right) .
\end{aligned}
$$

We deduce the more accurate inequality

$$
\ell_{1}(3 / 8) \geq 0.9375|\mathcal{B}|-2.1008878 M-2 \ell+o(1):=x_{1}-2 \ell .
$$

Example 4.2. The inequality (4.4) is obtained by studying the function

$$
\varphi(t)= \begin{cases}1 & \text { if } 0 \leq t \leq 5 / 8 \\ 1+\frac{4 \pi}{3} \sin \left(\frac{8 \pi}{3}(t-1)\right) & \text { if } 5 / 8 \leq t \leq 1\end{cases}
$$


The constant 3.893 is an approximate value of the sum

$$
\sum_{n=1}^{\infty}\left|\frac{32 \cos (5 \pi n / 8)}{9 n^{2}-16}\right|
$$

and could be replaced by the more accurate value 3.89284896 . We deduce a more convenient form of (4.4):

$$
\ell_{2}(3 / 8) \geq 0.2387324|\mathcal{B}|-0.9293493 M+o(1):=x_{3} .
$$

Example 4.3. The inequality (4.5) is obtained by studying the function $\varphi=$ $-w_{1,3 / 32,7 / 32,7 / 32}-\frac{1}{2} w_{1,0,1 / 16,7 / 16}$ with the notation of section 6 . Using the calculations detailed there, we get $\widehat{\varphi}(0)=-11 / 32$ and

$$
\begin{aligned}
\widehat{\varphi}(n)=\frac{4}{\pi^{2} n^{2}} \exp \left(\frac{7 i \pi n}{16}\right)\left(\cos \left(\frac{7 \pi n}{16}\right)-\cos \left(\frac{3 \pi n}{16}\right)\right) \\
+\frac{4}{\pi^{2} n^{2}} \exp \left(\frac{7 i \pi n}{8}\right)\left(\cos \left(\frac{\pi n}{8}\right)-1\right) .
\end{aligned}
$$

We deduce the more accurate inequality

$$
\ell_{2}(3 / 8) \geq 0.6875|\mathcal{B}|-2.1472415 M-\ell+o(1):=x_{4}-\ell .
$$

\section{Example 4.4. Consider}

$$
\varphi(t)= \begin{cases}0 & \text { if } t \in[0,3 / 8], \\ -8 x+3 & \text { if } t \in[3 / 8,1 / 2], \\ -1 & \text { if } t \in[1 / 2,7 / 8], \\ 8 x-8 & \text { if } t \in[7 / 8,1] .\end{cases}
$$

With the notation of section 6 , we have $\varphi=-w_{1,3 / 16,5 / 16,5 / 16}$. Using the calculations detailed there, we get $\widehat{\varphi}(0)=-1 / 2$ and

$$
\|\varphi\|-|\widehat{\varphi}(0)|=8 \sum_{n=1}^{\infty} \frac{|\cos (5 \pi n / 8)-\cos (3 \pi n / 8)|}{\pi^{2} n^{2}}<0.923879532512 .
$$

Since $\min _{[0,3 / 8]} \varphi=0, \min _{[3 / 8,1 / 2]} \varphi=\min _{[1 / 2,1]} \varphi=-1$, we obtain

$$
\ell_{1}(3 / 8) \geq \frac{|\mathcal{B}|}{2}-0.9238796 M-\ell+o(1):=x_{2}-\ell .
$$

Let us now explain how to deduce a bound for $\sigma$ from inequalities (4.6-4.9) and Lemma 3.1. Without loss of generality, we may assume that $|\mathcal{B}| / M \geq 3.9$; the inequality $|\mathcal{B}|<3.9 M$ would give $\sigma<0.4672$, a new record. Note that in this case, we have $x_{3} \geq 0$. We deduce from (4.6-4.9) the inequality

$$
M^{2} \geq \ell^{2}+2 \max \left(x_{1}-2 \ell, x_{2}-\ell\right) \max \left(x_{3}, x_{4}-\ell\right) .
$$

Put $L_{1}=x_{1}-x_{2}$ and $L_{2}=x_{4}-x_{3}$. One easily checks that $0 \leq L_{1} \leq L_{2}$ using the assumption $|\mathcal{B}|<3.9 M$. Let us simplify (4.10) according to the possible values of $\ell$.

For $\ell \leq L_{1}$, we get

$$
\begin{aligned}
M^{2} & \geq \ell^{2}+2\left(x_{1}-2 \ell\right)\left(x_{4}-\ell\right)=\left(2 x_{4}+x_{1}-\ell\right)^{2}-\left(2 x_{4}+x_{1}\right)^{2}+2 x_{1} x_{4} \\
& \geq L_{1}^{2}+2\left(x_{1}-2 L_{1}\right)\left(x_{4}-2 L_{1}\right),
\end{aligned}
$$

since $2 x_{4}+x_{1}-\ell \geq 2 x_{4}+x_{1}-L_{1} \geq 0$ for $|\mathcal{B}|>2.7832 M$. 
For $\ell \geq L_{2}$, we get

$$
M^{2} \geq \ell^{2}+2 x_{3}\left(x_{2}-\ell\right)=\left(\ell-x_{3}\right)^{2}-x_{3}^{2}-2 x_{2} x_{3} \geq L_{2}^{2}+2 x_{3}\left(x_{2}-L_{2}\right),
$$

since $\ell-x_{3} \geq L_{2}-x_{3} \geq 0$ for $|\mathcal{B}|>1.3738 M$.

For $L_{1} \leq \ell \leq L_{2}$, we get

$$
\begin{aligned}
M^{2} & \geq \ell^{2}+2\left(x_{2}-\ell\right)\left(x_{4}-\ell\right)=\left(x_{2}+x_{4}-\ell\right)^{2}-\left(x_{2}+x_{4}\right)^{2}+2 x_{2} x_{4} \\
& \geq L_{2}^{2}+2\left(x_{2}-L_{2}\right)\left(x_{4}-L_{2}\right),
\end{aligned}
$$

since $x_{2}+x_{4}-\ell \geq x_{2}+x_{4}-L_{2} \geq 0$ for $|\mathcal{B}|>2.5087 M$.

We thus get that, for any value of $\ell$, we have

$$
M^{2} \geq L_{2}^{2}+2\left(x_{2}-L_{2}\right)\left(x_{4}-L_{2}\right) .
$$

This inequality may be rewritten as

$$
0.2258540264|\mathcal{B}|^{2}-1.047945832|\mathcal{B}| M-0.06321968572 M^{2} \leq 0,
$$

which gives $|\mathcal{B}|<4.6994881 M$ and therefore $\sigma \leq 0.4773604$. The same method applied without using Example 4.4 leads to Li An-Ping's result $\sigma<0.4774024$.

We focused in this section on the case $\theta=3 / 8$. The examples presented here may be adapted to other values of $\theta$. However, it seems quite unlikely to obtain a significant improvement on $\sigma$ by choosing another special value of $\theta$. We made very few numerical experiments with other values of $\theta$, since it requires much work to get interesting inequalities, and our results were quite disappointing. It would be interesting to understand why the choice $\theta=3 / 8$ is almost optimal.

\section{USING DOUble Fourier SERIES}

When we are dealing with usual Fourier series, we basically have to square the functions to be able to use the 2-basis hypothesis. Using double Fourier series will enable us to directly use this condition. We can also use multiple Fourier series, but the computation will reduce them to double ones. However using $h$-tuple Fourier series would be interesting in the study of $h$-basis.

We proceed as in section 3 and introduce an auxiliary function $\varphi$ defined by

$$
\varphi\left(t_{1}, t_{2}\right)=\sum_{k_{1}, k_{2} \in \mathbb{Z}} \widehat{\varphi}\left(k_{1}, k_{2}\right) \exp \left(2 i \pi\left(k_{1} t_{1}+k_{2} t_{2}\right)\right)
$$

with $\|\varphi\|=\sum_{k_{1}, k_{2}}\left|\widehat{\varphi}\left(k_{1}, k_{2}\right)\right|<\infty$. As before we have

$$
S:=\sum_{b_{1} b_{2} \in \mathcal{B}} \varphi\left(\frac{b_{1}}{N}, \frac{b_{2}}{N}\right)=\sum_{k_{1}, k_{2} \in \mathbb{Z}} \widehat{\varphi}\left(k_{1}, k_{2}\right) f_{\mathcal{B}}\left(\frac{k_{1}}{N}\right) f_{\mathcal{B}}\left(\frac{k_{2}}{N}\right) .
$$

We shall use the following quantities, defined by Güntürk and Nathanson [2]:

$$
\begin{gathered}
C_{\text {axial }}=\sum_{\substack{k \in \mathbb{Z} \\
k \neq 0}}(|\widehat{\varphi}(k, 0)|+|\widehat{\varphi}(0, k)|) \quad \text { and } C_{\text {main }}=\sum_{\substack{k_{1}, k_{2} \in \mathbb{Z} \\
k_{1} k_{2} \neq 0}}\left|\widehat{\varphi}\left(k_{1}, k_{2}\right)\right|, \\
\alpha_{1}=\min _{\substack{t_{1}, t_{2} \in[0,1] \\
t_{1}+t_{2} \leq 1}} \varphi\left(t_{1}, t_{2}\right) \quad \text { and } \alpha_{2}=\min _{\substack{t_{1}, t_{2} \in[0,1] \\
t_{1}+t_{2} \geq 1}} \varphi\left(t_{1}, t_{2}\right), \\
L=\sum_{n \geq N} r_{\mathcal{B}}(n) .
\end{gathered}
$$


The one-dimensional case studied in section 3 may be easily extended. The proof of Lemma 3.1 implies

$$
L \leq M^{2}
$$

and we deduce from (5.1) inequalities analogous to (3.4) and (3.5):

$$
\left(|\mathcal{B}|^{2}-L\right) \alpha_{1}+L \alpha_{2} \leq S \leq \widehat{\varphi}(0,0)|\mathcal{B}|^{2}+C_{\text {axial }}|\mathcal{B}| M+C_{\text {main }} M^{2}+o\left(|\mathcal{B}|^{2}\right)
$$

For $\alpha_{1} \geq \alpha_{2}$ we can plug (5.2) into (5.3) to get

$$
0 \leq\left(\widehat{\varphi}(0,0)-\alpha_{1}\right)|\mathcal{B}|^{2}+C_{\text {axial }}|\mathcal{B}| M+\left(C_{\text {main }}+\alpha_{1}-\alpha_{2}\right) M^{2}+o\left(|\mathcal{B}|^{2}\right)
$$

This inequality involves a second-degree polynomial in $M /|\mathcal{B}|$, whose leading coefficient is positive and whose roots have opposite signs when $\widehat{\varphi}(0,0)-\alpha_{1} \leq 0$. Note that this last condition also implies the condition $\alpha_{1} \geq \alpha_{2}$, as in the one-dimensional case. Computing the roots of this polynomial leads to the estimate

$$
\frac{M}{|\mathcal{B}|} \geq \frac{-C_{\text {axial }}+\sqrt{C_{\text {axial }}^{2}+4\left(\alpha_{1}-\widehat{\varphi}(0,0)\right)\left(C_{\text {main }}+\alpha_{1}-\alpha_{2}\right)}}{2\left(C_{\text {main }}+\alpha_{1}-\alpha_{2}\right)}+o(1),
$$

from which we deduce the following theorem.

Theorem 5.1. For $\alpha_{1} \geq \widehat{\varphi}(0,0)$, we have

$$
\sigma \leq \frac{1}{2}-\frac{1}{2}\left(\frac{-C_{a x i a l}+\sqrt{C_{a x i a l}^{2}+4\left(\alpha_{1}-\widehat{\varphi}(0,0)\right)\left(C_{m a i n}+\alpha_{1}-\alpha_{2}\right)}}{2\left(C_{\text {main }}+\alpha_{1}-\alpha_{2}\right)}\right)^{2}
$$

Note that the estimate given in this theorem is invariant when $\varphi$ is replaced by $A \varphi+B$, for any constants $A$ and $B>0$.

Let us now study several examples. It is quite natural to require that $\varphi$ is constant, say 0 , in the triangle $t_{1}, t_{2}, 1-t_{1}-t_{2} \geq 0$. Since $\varphi$ has to be doubly 1 -periodical, this implies that $\varphi\left(1, t_{2}\right)=\varphi\left(t_{1}, 1\right)=\varphi(t, 1-t)=0$. The first three examples are simple functions $\varphi$ satisfying these boundaries conditions and are two-dimensional extensions of Examples 3.2 and 3.3.

Example 5.1. Define $\varphi$ on $[0,1]^{2}$ by

$$
\varphi\left(t_{1}, t_{2}\right)= \begin{cases}0 & \text { if } t_{1}+t_{2} \leq 1 \\ \sin \left(\pi t_{1}\right) \sin \left(\pi t_{2}\right) \sin \left(\pi\left(t_{1}+t_{2}\right)\right) & \text { if } t_{1}+t_{2} \geq 1\end{cases}
$$

We get $\alpha_{1}=0$, and one easily checks that $\alpha_{2}=\varphi(2 / 3,2 / 3)=-(\sqrt{3} / 2)^{3}$. The Fourier coefficients also enjoy the symmetry property $\widehat{\varphi}(r, s)=\widehat{\varphi}(s, r)$ and are 
given by

$$
\widehat{\varphi}(r, s)= \begin{cases}0 & \text { for } r\left(r^{2}-1\right) s\left(s^{2}-1\right)(r-s)(r-s+1)(r-s-1) \neq 0, \\ \frac{1}{8 \pi(r-1)(r+1)} & \text { for } r=s \neq \pm 1 \\ -\frac{1}{16 \pi r(r-1)} & \text { for } r=s+1 \neq 0,1 \\ -\frac{1}{16 \pi r(r+1)} & \text { for } r=s-1 \neq 0,-1, \\ -\frac{1}{16 \pi r(r-1)} & \text { for } s=1 \text { and } r \neq 0,1 \\ -\frac{1}{16 \pi r(r+1)} & \text { for } s=-1 \text { and } r \neq 0,-1 \\ \frac{1}{8 \pi(r-1)(r+1)} & \text { for } s=0 \text { and } r \neq \pm 1, \\ -\frac{1}{16 \pi} & \text { for } r=-s= \pm 1, \\ \frac{3-2 i \pi}{32 \pi} & \text { for }(r, s) \in\{(1,1),(0,-1)\} \\ \frac{3+2 i \pi}{32 \pi} & \text { for }(r, s) \in\{(-1,-1),(0,1)\} \\ -\frac{3}{8 \pi} & \text { for } r=s=0 .\end{cases}
$$

For all $(r, s) \in \mathbb{Z}^{2} \backslash\{(1,1),(0,-1),(-1,-1),(0,1)\}$, the sign of $\widehat{\varphi}(r, s)$ is obvious, and the above formulas make $C_{\text {main }}$ and $C_{\text {axial }}$ computable:

$$
C_{a x i a l}=4 \sum_{r \geq 2} \frac{1}{8 \pi(r-1)(r+1)}+4 \frac{\sqrt{9+4 \pi^{2}}}{32 \pi}=\frac{3+\sqrt{9+4 \pi^{2}}}{8 \pi}
$$

and

$$
\begin{aligned}
C_{\text {main }} & =\sum_{r \geq 2} \frac{2}{8 \pi\left(r^{2}-1\right)}+\sum_{r \geq 2} \frac{4}{16 \pi r(r-1)}+\frac{2}{16 \pi}+\frac{2 \sqrt{9+4 \pi^{2}}}{32 \pi}+\sum_{r \geq 3} \frac{8}{16 \pi r(r-1)} \\
& =\frac{13+\sqrt{9+4 \pi^{2}}}{16 \pi} .
\end{aligned}
$$

Plugging in these values in Theorem 5.1 gives the bound $\sigma<0.48069$.

Example 5.2. Define $\varphi$ on $[0,1]^{2}$ by

$$
\varphi\left(t_{1}, t_{2}\right)= \begin{cases}0 & \text { if } t_{1}+t_{2} \leq 1 \\ \left(1-t_{1}\right)\left(1-t_{2}\right)\left(1-t_{1}-t_{2}\right) & \text { if } t_{1}+t_{2} \geq 1\end{cases}
$$

We thus get $\alpha_{1}=0$, and an easy calculation shows that $\alpha_{2}=\varphi(2 / 3,2 / 3)=-1 / 27$. The Fourier coefficients enjoy the symmetry property $\widehat{\varphi}(r, s)=\widehat{\varphi}(s, r)$ and are given by

$$
\widehat{\varphi}(r, s)= \begin{cases}-\frac{r^{2}-r s+s^{2}}{8 \pi^{4} r^{2} s^{2}(r-s)^{2}} & \text { for } r s(r-s) \neq 0, \\ \frac{\pi^{2} r^{2}+3 i \pi r-6}{24 \pi^{4} r^{4}} & \text { for } r=s \neq 0, \\ \frac{\pi^{2} r^{2}-3 i \pi r-6}{24 \pi^{4} r^{4}} & \text { for } s=0 \neq r, \\ -\frac{1}{120} & \text { for } r=s=0 .\end{cases}
$$

Since

$$
\begin{aligned}
\sum_{r s(r-s) \neq 0} \frac{2\left(r^{2}-r s+s^{2}\right)}{r^{2} s^{2}(r-s)^{2}} & =\sum_{r s(r-s) \neq 0}\left(\frac{1}{r^{2} s^{2}}+\frac{1}{r^{2}(r-s)^{2}}+\frac{1}{s^{2}(r-s)^{2}}\right) \\
& =3 \sum_{r s(r-s) \neq 0} \frac{1}{r^{2} s^{2}}=3 \sum_{r s \neq 0} \frac{1}{r^{2} s^{2}}-3 \sum_{r \neq 0} \frac{1}{r^{4}}=\frac{4 \pi^{4}}{15},
\end{aligned}
$$


we get

$$
C_{\text {main }}=\frac{1}{60}+S \quad \text { and } \quad C_{\text {axial }}=2 S
$$

with

$$
S=\sum_{r=1}^{\infty} \frac{\sqrt{\pi^{4} r^{4}-3 \pi^{2} r^{2}+36}}{12 \pi^{4} r^{4}} \in[0.014082,0.01408201] .
$$

Plugging in these values in Theorem 5.1 gives the bound $\sigma<0.48004$.

Example 5.3. Define $\varphi$ on $[0,1]^{2}$ by

$$
\varphi\left(t_{1}, t_{2}\right)= \begin{cases}0 & \text { if } t_{1}+t_{2} \leq 1 \\ 1+\cos \left(\pi t_{1}\right)+\cos \left(\pi t_{2}\right)+\cos \left(\pi\left(t_{1}+t_{2}\right)\right) & \text { if } t_{1}+t_{2} \geq 1\end{cases}
$$

As in the previous examples, we get $\alpha_{1}=0$ and $\alpha_{2}=\varphi(2 / 3,2 / 3)=-1 / 2$. The Fourier coefficients also enjoy the symmetry property $\widehat{\varphi}(r, s)=\widehat{\varphi}(s, r)$ and are given by

$$
\widehat{\varphi}(r, s)= \begin{cases}\frac{-2\left(4 r^{2}-4 r s+4 s^{2}-3\right)}{\pi^{2}\left(4 r^{2}-1\right)\left(4 s^{2}-1\right)\left(4(r-s)^{2}-1\right)} & \text { for } r s \neq 0, \\ \frac{8 r^{3}-6 r-i \pi\left(4 r^{2}-1\right) / 2}{\pi^{2} r\left(4 r^{2}-1\right)^{2}} & \text { for } r \neq s=0, \\ \frac{\pi^{2}-12}{2 \pi^{2}} & \text { for } r=s=0 .\end{cases}
$$

Since $\widehat{\varphi}(r, s) \geq 0$ for $r s(r-s) \neq 0$ and $\widehat{\varphi}(r, r) \leq 0$ for $r \neq 0$, we find

$$
\begin{aligned}
C_{\text {main }} & =-\sum_{r s \neq 0} \widehat{\varphi}(r, s)+2 \sum_{r \neq 0} \widehat{\varphi}(r, r) \\
& =-\varphi(0,0)+\widehat{\varphi}(0,0)+\sum_{r \neq 0}(\widehat{\varphi}(r, 0)+\widehat{\varphi}(0, r)+2 \widehat{\varphi}(r, r)) \\
& =\frac{\pi^{2}-12}{2 \pi^{2}}+\frac{16}{\pi^{2}} \sum_{r \geq 1} \frac{4 r^{2}-3}{\left(4 r^{2}-1\right)^{2}}=\frac{\pi^{2}-12}{2 \pi^{2}}+\frac{16}{\pi^{2}}\left(\frac{3}{2}-\frac{\pi^{2}}{8}\right)=\frac{3\left(12-\pi^{2}\right)}{2 \pi^{2}} .
\end{aligned}
$$

Computing the first 300000 terms of the series defining $C_{\text {axial }}$ gives the estimate $C_{\text {axial }} \in[0.362208,0.362209]$. Plugging in these values in Theorem 5.1 gives the bound $\sigma<0.47927$.

Note that

$$
1+\cos \left(\pi t_{1}\right)+\cos \left(\pi t_{2}\right)+\cos \left(\pi\left(t_{1}+t_{2}\right)\right)=4 \cos \left(\frac{\pi t_{1}}{2}\right) \cos \left(\frac{\pi t_{2}}{2}\right) \cos \left(\frac{\pi\left(t_{1}+t_{2}\right)}{2}\right),
$$

and we see that Examples 5.1-5.3 correspond to the cases $(a, b, c)=(\pi, \pi, \pi),(0,0,0)$, $(\pi / 2, \pi / 2, \pi / 2)$ respectively, in the family of functions

$$
\varphi_{a, b, c}\left(t_{1}, t_{2}\right)= \begin{cases}1 & \text { if } t_{1}+t_{2} \leq 1 \\ \sin \left(a\left(1-t_{1}\right)\right) \sin \left(b\left(1-t_{2}\right)\right) \sin \left(c\left(1-t_{1}-t_{2}\right)\right) & \text { if } t_{1}+t_{2} \geq 1\end{cases}
$$

It would be interesting to find the optimal choice for the triple of complex numbers $(a, b, c)$.

We can also add extra factors to get good bounds, as in the next example.

Example 5.4 ([2]). Define $\varphi$ on $[0,1]^{2}$ by

$$
\varphi\left(t_{1}, t_{2}\right)= \begin{cases}1 & \text { if } t_{1}+t_{2} \leq 1 \\ 1-40\left(1-t_{1}\right)\left(1-t_{2}\right)\left(1-\left(2-t_{1}-t_{2}\right)^{6}\right) & \text { if } t_{1}+t_{2} \geq 1\end{cases}
$$


Güntürk and Nathanson [2] computed $\alpha_{1}=1$ and $\alpha_{2}=1-15 \times 2^{-5 / 3}$, and gave the estimates $2.90278 \leq C_{\text {axial }} \leq 2.90289$ and $4.75145 \leq C_{\text {main }} \leq 4.76146$. Plugging these values in Theorem 5.1 gives the upper bound $\sigma<0.4789$.

Let us now explain how Lemma 4.1 can also be used here to sharpen these results. Put $\ell_{1}^{0}=0.2387234|\mathcal{B}|-0.9293493 M$ and $\ell_{2}^{0}=0.5|\mathcal{B}|-1.9238796 M$. From Lemma 4.1 and formulas (4.7) and (4.9), we get

$$
M^{2}-\ell^{2} \geq 2 \ell_{1}(3 / 8) \ell_{2}(3 / 8) \geq 2 \ell_{1}^{0} \ell_{2}^{0} .
$$

Also define

$$
\alpha_{3}=\min _{\substack{\left(t_{1}, t_{2}\right) \in[0,1]^{2} \backslash[1 / 2,1]^{2} \\ t_{1}+t_{2} \geq 1}} \varphi\left(t_{1}, t_{2}\right)
$$

The left-hand side of (5.3) may be sharpened as $S \geq\left(|\mathcal{B}|^{2}-L\right) \alpha_{1}+\ell^{2} \alpha_{2}+\left(L-\ell^{2}\right) \alpha_{3}$, from which we deduce a refinement of (5.4):

$$
\begin{aligned}
\alpha_{1}\left(|\mathcal{B}|^{2}-M^{2}\right)+\alpha_{2} M^{2}+2\left(\alpha_{3}-\right. & \left.\alpha_{2}\right) \ell_{1}^{0} \ell_{2}^{0} \\
& \leq \widehat{\varphi}(0,0)|\mathcal{B}|^{2}+C_{\text {axial }}|\mathcal{B}| M+C_{\text {main }} M^{2}+o\left(|\mathcal{B}|^{2}\right) .
\end{aligned}
$$

This last inequality still leads to a second degree inequality in $|\mathcal{B}| / M$. We find in this way the following estimates:

$\begin{array}{cccc}\text { Example } & \alpha_{3} & |\mathcal{B}| / M & \sigma \\ 5.1 & -1 / 2 & 5.0040621 & 0.48004 \\ 5.2 & -1 / 32 & 4.9765891 & 0.47982 \\ 5.3 & 1-\sqrt{2} & 4.8875498 & 0.47907 \\ 5.4 & -3.4546435221 & 4.8474023 & 0.47873\end{array}$

\section{USING PERIODS GREATER THAN ONE}

We shall focus on the case $t_{k}=k / p$ for some period $p>1$, and more precisely we shall study the following family of functions.

Let $p, \alpha$ and $\beta$ be real numbers with $0 \leq \alpha<\beta \leq p / 2$ and $p \geq 1$. Define a $p$-periodic real function $w_{p, \alpha, \beta}$ by

$$
w_{p, \alpha, \beta}(x)= \begin{cases}1 & \text { if }|x| \leq \alpha \\ 1-\frac{|| x|-\alpha|}{\beta-\alpha} & \text { if } \alpha \leq|x| \leq \beta \\ 0 & \text { if } \beta \leq|x| \leq p / 2\end{cases}
$$

Since $w_{p, \alpha, \beta}$ is $p$-periodic, it is natural to look for its Fourier expansion.

Lemma 6.1. We have $\widehat{w_{p, \alpha, \beta}}(0)=(\alpha+\beta) / p$ and

$$
\widehat{w_{p, \alpha, \beta}}(n)=\frac{p}{2 \pi^{2} n^{2}(\beta-\alpha)}\left(\cos \left(\frac{2 \pi n \alpha}{p}\right)-\cos \left(\frac{2 \pi n \beta}{p}\right)\right),
$$

for $n \neq 0$. 
Proof. The evaluation of $\widehat{w_{p, \alpha, \beta}}(0)$ easily follows from the formula from a trapezoid. Let $n$ be a nonzero integer. We get

$$
\begin{aligned}
\widehat{w_{p, \alpha, \beta}}(n) & =\frac{1}{p} \int_{-p / 2}^{p / 2} w_{p, \alpha, \beta}(x) \exp (-2 i \pi n x / p) d x \\
& =\frac{1}{2 i \pi n} \int_{-p / 2}^{p / 2} w_{p, \alpha, \beta}^{\prime}(x) \exp (-2 i \pi n x / p) d x \\
& =\frac{1}{2 i \pi n(\beta-\alpha)}\left(\int_{-\beta}^{-\alpha} \exp (-2 i \pi n x / p) d x-\int_{\alpha}^{\beta} \exp (-2 i \pi n x / p) d x\right) \\
& =\frac{1}{\pi n(\beta-\alpha)} \int_{\alpha}^{\beta} \sin (2 \pi n x / p) d x,
\end{aligned}
$$

and the lemma follows.

Note that $\widehat{w_{p, 0, \beta}}(n) \geq 0$ and $\left\|w_{p, 0, \beta}\right\|=w_{p, 0, \beta}(0)=1$. This is the only obvious case of nonnegativity. This special case has been studied by $\mathrm{Yu}$ [9], and he proved the following lemma.

Lemma 6.2. We have

$$
D_{\mathcal{B}}\left(w_{p, 0, \beta}\right) \leq D_{\mathcal{B}}\left(w_{1,0, \beta}\right) \leq|B|^{2}-(1-\beta) 2 N+o(N) .
$$

For $\kappa \in \mathbb{R}$, let us introduce the $p$-periodic real function $w_{p, \alpha, \beta, \kappa}$ defined by $w_{p, \alpha, \beta, \kappa}(x)=w_{p, \alpha, \beta}(x+\kappa)$. Let us estimate $R_{\mathcal{B}}\left(w_{p, \alpha, \beta, \kappa}\right)$. By Lemma 2.3 we obtain

$$
R_{\mathcal{B}}\left(w_{p, \alpha, \beta, \kappa}\right) \leq 2 N I_{\kappa}+|\mathcal{B}|^{2}-2 N+o(N),
$$

with $I_{\kappa}=\int_{0}^{1} w_{p, \alpha, \beta, \kappa}(x) d x=\int_{\kappa}^{\kappa+1} w_{p, \alpha, \beta}(x) d x$. Using formula (2.4) and the fact that $\widehat{w_{p, \alpha, \beta}, \kappa}(n)=\exp (2 i \pi n \kappa / p) \widehat{w_{p, \alpha, \beta}}(n)$, we get

$$
R_{\mathcal{B}}\left(w_{p, \alpha, \beta, \kappa}\right) \geq \frac{\alpha+\beta}{p}|\mathcal{B}|^{2}-\sum_{n \neq 0}\left|\widehat{w_{p, \alpha, \beta}}(n)\right| \times\left|f_{\mathcal{B}}\left(\frac{n}{p N}\right)\right|^{2} .
$$

Remark that the right-hand side of (6.2) does not depend on $\kappa$. So we need to minimize $I_{\kappa}$ to get the lowest possible upper bound in (6.1). It is easy to check that $I_{\kappa}$ is minimal for $\kappa=(p-1) / 2$. More precisely, we have

$$
\min _{\kappa} I_{\kappa}= \begin{cases}0 & \text { if } p \geq 1+2 \beta, \\ \frac{(\beta-(p-1) / 2)^{2}}{\beta-\alpha} & \text { if } 1+2 \alpha \leq p \leq 1+2 \beta, \\ \beta+\alpha-(p-1) & \text { if } p \leq 1+2 \alpha .\end{cases}
$$

The main difficulty is thus to estimate the right-hand side of (6.2). Let us describe several approaches, including that of $\mathrm{Yu}[9$. Since the case when $\widehat{w}(n) \geq 0$ is much easier to handle and occurs when $\alpha=0$, all these methods link cases with $\alpha \neq 0$ to other cases with $\alpha=0$. Let us start with this main special case.

Example 6.1. The case $\alpha=0$.

In this case $(6.2)$ becomes

$$
R_{\mathcal{B}}\left(w_{p, 0, \beta, \kappa}\right) \geq \frac{2 \beta}{p}|\mathcal{B}|^{2}-D_{\mathcal{B}}\left(w_{p, 0, \beta}\right) \geq\left(\frac{2 \beta}{p}-1\right)|\mathcal{B}|^{2}+(1-\beta) 2 N+o(N),
$$


by Lemma 6.2 . We deduce from this last inequality and (6.1) the bound

$$
\frac{2 N}{|\mathcal{B}|^{2}} \leq \frac{2(1-\beta / p)}{2-\beta-I_{\kappa}}+o(1)
$$

We use (6.3) to get upper bounds for $\sigma$. The choice of parameters $\beta=0.283654$, $p=1.390814$ leads to $\sigma<0.471345691842$.

Example 6.2. The case $\beta=3 \alpha$.

This case enjoys so many properties that we can describe several approaches.

6.2.1. A first inequality. First notice the basic equality

$$
\cos x-\cos 3 x=2 \cos x(1-\cos 2 x) .
$$

By Lemma 6.1, it implies the inequality

$$
\left|\widehat{w_{p, \alpha, 3 \alpha, \kappa}}(n)\right| \leq 2 \widehat{w_{p, 0,2 \alpha}}(n) .
$$

Plugging (6.5) into (6.2) gives

$$
\begin{aligned}
R_{\mathcal{B}}\left(w_{p, \alpha, 3 \alpha, \kappa}\right) & \geq \frac{4 \alpha}{p}|\mathcal{B}|^{2}-2\left(D_{\mathcal{B}}\left(w_{p, 0,2 \alpha}\right)-\widehat{w_{p, 0,2 \alpha}}(0)|\mathcal{B}|^{2}\right) \\
& \geq\left(-2+\frac{8 \alpha}{p}\right)|\mathcal{B}|^{2}+(1-2 \alpha) 4 N+o(N),
\end{aligned}
$$

by Lemma 6.2. We use Lemma 6.1 to get the bound

$$
\frac{2 N}{|\mathcal{B}|^{2}} \leq \frac{3-8 \alpha / p}{3-4 \alpha-I_{\kappa}}+o(1)
$$

By (6.3) this provides upper bounds for $\sigma$. The choice of parameters $\alpha=0.08944$, $p=1.3992$ leads to $\sigma<0.4756831656$.

6.2.2. A refined inequality. The inequality (6.5) may be refined when $n \alpha / p$ only takes a finite number of values modulo 1 . For instance, let us consider the case $p=2 q \alpha$, for some positive integer $q$. We obtain

$$
\left|w_{2 q \alpha, \alpha, 3 \alpha, \kappa}(n)\right| \leq 2 \cos \left(\frac{\pi}{q}\right) \widehat{w_{2 q \alpha, 0,2 \alpha}}(n) .
$$

We proceed as below to find

$$
\frac{2 N}{|\mathcal{B}|^{2}} \leq \frac{1-2 / q+(1-1 / q) 2 \cos (\pi / q)}{1-I_{\kappa}+(1-2 \alpha) 2 \cos (\pi / q)}+o(1) .
$$

We use (6.3) to get upper bounds for $\sigma$. Studying the denominator in terms of $\alpha$ gives the best choice of parameter: $\alpha_{0}=\left(4(q-3)^{2}+32 \cos (\pi / q)\right)^{-1 / 2}$. For $q=6$ this leads to $\sigma<0.471719568373027$, while the case $q=7$ gives $\sigma<0.471917965505613$.

6.2.3. A special inequality. We can further refine the preceding approach. Let us focus on the case $p=12 \alpha$. Put $x_{n}=2 \pi n \alpha / p=\pi n / 6$. We check that

$$
\left|\cos x_{n}-\cos 3 x_{n}\right| \leq 1-\cos 2 x_{n}+\frac{\sqrt{3}-1}{2}\left(1-\cos 3 x_{n}\right),
$$

which may be restated as

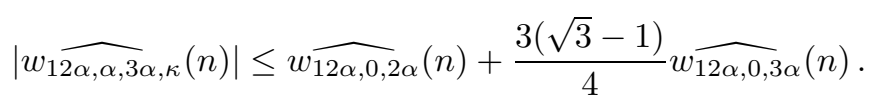


Plugging (6.2) into this last estimate, we obtain

$R\left(w_{12 \alpha, \alpha, 3 \alpha, \kappa}\right) \geq \frac{|\mathcal{B}|^{2}}{3}-\frac{5}{6}|\mathcal{B}|^{2}-\frac{9(\sqrt{3}-1)}{16}|\mathcal{B}|^{2}+(1-2 \alpha) 2 N+\frac{3(\sqrt{3}-1)}{4}(1-3 \alpha) 2 N$,

from which we deduce

$$
\sigma \leq \frac{3 / 2+9(\sqrt{3}-1) / 16}{2(1-\alpha)+3(\sqrt{3}-1)(1-3 \alpha) / 4-I_{\kappa}} .
$$

The best choice for $\alpha$ is $(34+18 \sqrt{3})^{-1 / 2}$, and it gives $\sigma<0.470711552467$.

6.2.4. Yu's approach 9]. First note that the value of $R_{\mathcal{B}}\left(w_{p, \alpha, \beta, \kappa}\right)$ only depends on the values of $w$ on $[0,2]$. So we only need to connect $w$ to functions of type $w_{p, 0, \beta, \kappa}$ on this interval. The case $\beta=3 \alpha$ is of special interest since we have $w_{p, \alpha, 3 \alpha}=w_{p, 0,2 \alpha, \alpha}+w_{p, 0,2 \alpha,-\alpha}$ on the interval $[-3 \alpha, 3 \alpha]$. In this formula, all the periods are chosen to be the same. However, it gives better results to deal with different periods, basically because $1 /(p-\eta)+1 /(p+\eta)>2 / p$, for any $\eta \in] 0, p[$.

More precisely, $\mathrm{Yu}$ [9] considered the function $w=w_{p_{1}, 0,2 \alpha, \kappa_{1}}+w_{p_{2}, 0,2 \alpha, \kappa_{2}}$ with $2 \alpha=0.2257, p_{1}=1.275, p_{2}=1.5889, \kappa_{1}=0.1375$ and $\kappa_{2}=0.2257$. It is defined on $[0,2]$ by

$$
w(t)= \begin{cases}(0.0882-t) / 0.2257 & \text { if } t \in[0,0.0882], \\ 0 & \text { if } t \in[0.0882,0.9118], \\ (t-0.9118) / 0.2257 & \text { if } t \in[0.9118,1.1375], \\ 1 & \text { if } t \in[1.1375,1.3632], \\ (1.5889-t) / 0.2257 & \text { if } t \in[1.3632,1.5889], \\ 0 & \text { if } t \in[1.5889,2] .\end{cases}
$$

By (6.1) we have the upper bound $R_{\mathcal{B}}(w) \leq 2 N I+|\mathcal{B}|^{2}-2 N+o(N)$ with $I=$ $0.0882^{2} / 0.2257$. Combining (6.2) with Lemma 6.2 provides the lower bounds

$$
\begin{aligned}
R_{\mathcal{B}}(w) & \geq 4 \alpha\left(\frac{1}{p_{1}}+\frac{1}{p_{2}}\right)|\mathcal{B}|^{2}-D_{\mathcal{B}}\left(w_{p_{1}, 0,2 \alpha, \kappa_{1}}\right)-D_{\mathcal{B}}\left(w_{p_{2}, 0,2 \alpha, \kappa_{2}}\right)+o(N) \\
& \geq 4 \alpha\left(\frac{1}{p_{1}}+\frac{1}{p_{2}}\right)|\mathcal{B}|^{2}-2|\mathcal{B}|^{2}+2(1-2 \alpha) 2 N+o(N) .
\end{aligned}
$$

By putting these bounds together we obtain

$$
\frac{2 N}{|\mathcal{B}|^{2}} \leq \frac{3-4 \alpha\left(\frac{1}{p_{1}}+\frac{1}{p_{2}}\right)}{3-4 \alpha-I} .
$$

The choice of parameters given above proves the estimate $\sigma<0.46972$.

Example 6.3. Using Cauchy-Schwarz inequality.

We apply Cauchy-Schwarz inequality as follows: we first notice the equalities

$$
\begin{aligned}
\left|\widehat{w_{p, \alpha, \beta}}(n)\right| & =\frac{p}{2 \pi^{2} n^{2}(\beta-\alpha)}\left|\cos \left(\frac{2 \pi n \alpha}{p}\right)-\cos \left(\frac{2 \pi n \beta}{p}\right)\right| \\
& =\frac{p}{\pi^{2} n^{2}(\beta-\alpha)}\left|\sin \left(\frac{\pi n(\beta-\alpha)}{p}\right) \sin \left(\frac{\pi n(\beta+\alpha)}{p}\right)\right| \\
& =\sqrt{\frac{\beta+\alpha}{\beta-\alpha} \widehat{w_{p, 0, \beta+\alpha}}(n) \widehat{w_{p, 0, \beta-\alpha}}(n)}
\end{aligned}
$$


and then deduce the upper estimate

$$
\begin{aligned}
\sum_{n \neq 0}\left|\widehat{w_{p, \alpha, \beta}}(n)\right| \times\left|f_{\mathcal{B}}\left(\frac{n}{p N}\right)\right|^{2} \\
\quad \leq \sqrt{\frac{\beta+\alpha}{\beta-\alpha}} \sqrt{D_{\mathcal{B}}\left(w_{p, 0, \beta+\alpha}\right)-\frac{\beta+\alpha}{p}|\mathcal{B}|^{2}} \sqrt{D_{\mathcal{B}}\left(w_{p, 0, \beta-\alpha}\right)-\frac{\beta-\alpha}{p}|\mathcal{B}|^{2}} .
\end{aligned}
$$

Using formula (6.2) and Lemma 6.2 gives

$$
\begin{aligned}
R_{\mathcal{B}}\left(w_{p, \alpha, \beta, \kappa}\right) \geq \frac{\alpha+\beta}{p}|\mathcal{B}|^{2} & -\sqrt{\frac{\beta+\alpha}{\beta-\alpha}} \sqrt{\left(1-\frac{\beta+\alpha}{p}\right)|\mathcal{B}|^{2}-(1-\beta-\alpha) 2 N} \\
& \times \sqrt{\left(1-\frac{\beta-\alpha}{p}\right)|\mathcal{B}|^{2}-(1-\beta+\alpha) 2 N} .
\end{aligned}
$$

Combining (6.1), (6.3) and (6.6) leads to a second degree inequation in $2 N /|\mathcal{B}|^{2}$. The choice of parameters $(\alpha, \beta, p)=(0.27819,0.071547,1.398004)$ provides the better bound $\sigma \leq 0.469055014865$, a new record.

\section{REFERENCES}

[1] L. An-Ping, On 2-additive basis, http://www.peerevaluation.org/read/libraryID:28270try (2012).

[2] C. Sinan Güntürk and Melvyn B. Nathanson, A new upper bound for finite additive bases, Acta Arith. 124 (2006), no. 3, 235-255, DOI 10.4064/aa124-3-3. MR.2250418 (2007f:11012)

[3] Walter Klotz, Eine obere Schranke für die Reichweite einer Extremalbasis zweiter Ordnung (German), J. Reine Angew. Math. 238 (1969), 161-168. MR.0246848 (40 \#117)

[4] L. Moser, On the representation of $1,2, \cdots, n$ by sums, Acta Arith. 6 (1960), 11-13. MR.0122800(23 \#A133)

[5] L. Moser, J. R. Pounder, and J. Riddell, On the cardinality of h-bases for $n$, J. London Math. Soc. 44 (1969), 397-407. MR0238798 (39 \#162)

[6] Arnulf Mrose, Untere Schranken für die Reichweiten von Extremalbasen fester Ordnung (German), Abh. Math. Sem. Univ. Hamburg 48 (1979), 118-124, DOI 10.1007/BF02941296. MR537452(80g:10058)

[7] J. Riddell, On bases for sets of integers, Master's Thesis, University of Alberta, 1960.

[8] Hans Rohrbach, Ein Beitrag zur additiven Zahlentheorie (German), Math. Z. 42 (1937), no. 1, 1-30, DOI 10.1007/BF01160061. MR1545658

[9] Gang Yu, Upper bounds for finite additive 2-bases, Proc. Amer. Math. Soc. 137 (2009), no. 1, 11-18, DOI 10.1090/S0002-9939-08-09430-6. MR.2439419 (2009g:11010)

Centre de Recherches Mathématiques, CNRS UMi 3457, Université de Montréal, Case Postale 6128, Succursale Centre-Ville, Montréal, Quebec, Canada H3C 3J7 E-mail address: habsieger@CRM.UMontreal.ca 